\title{
ASYMPTOTIC BEHAVIOR OF SOLUTIONS \\ FOR A SEMIBOUNDED NONMONOTONE \\ EVOLUTION EQUATION
}

NIKOS KARACHALIOS, NIKOS STAVRAKAKIS,

AND PAVLOS XANTHOPOULOS

Received 3 September 2002

We consider a nonlinear parabolic equation involving nonmonotone diffusion. Existence and uniqueness of solutions are obtained, employing methods for semibounded evolution equations. Also shown is the existence of a global attractor for the corresponding dynamical system.

\section{Introduction}

We consider the following nonlinear parabolic initial boundary value problem in the open bounded interval $\Omega \subset \mathbb{R}$

$$
\begin{aligned}
u_{t}-a(u) u_{x x}-b(u) u_{x}^{2}-\lambda \sigma(u) & =f(x), \quad x \in \Omega, t>0, \\
u(x, 0) & =u_{0}(x), \\
\left.u\right|_{\partial \Omega} & =0, \quad t>0 .
\end{aligned}
$$

This problem extends the well studied porous medium diffusion, since no certain relationship between the coefficients $a(u)$ and $b(u)$ is assumed. Let us mention that special cases of this system may typically arise in plasma physics within the context of the fluid treatment of charged particles, and in density-dependent reaction diffusion processes in mathematical biology. Naturally enough, these systems imply only positive values for $u(x, t)$; however, in the following treatment, we do not impose such a restriction.

In order to demonstrate a specific case-modelled system, we consider the collisionless evolution equation for the electron pressure $P=n T$, which, if we ignore viscosity, gets the following form in the $x$-direction (see, Balescu [5])

$$
\frac{3}{2} P_{t}=-q_{x}-\frac{3}{2} u P_{x}-\frac{5}{2} P u_{x}
$$

Copyright (C) 2003 Hindawi Publishing Corporation Abstract and Applied Analysis 2003:9 (2003) 521-538 2000 Mathematics Subject Classification: 35K55, 35B40, 35B41

URL: http://dx.doi.org/10.1155/S1085337503210022 
where $u$ represents the electron velocity and $q$ is the heat flux. Now, applying Darcy's law (see, Aronson [3])

$$
u=-c P_{x}, \quad c>0
$$

to the above equation, we get

$$
\frac{3}{2} P_{t}=-q_{x}+\frac{3}{2} c P_{x}^{2}+\frac{5}{2} c P P_{x x}
$$

We see that the first term on the right-hand side corresponds to porous medium diffusion (not considered here), whereas the other two terms constitute a specific case of (1.1a), with $a(P)=(5 / 3) c P$ and $b(P)=c$.

Concerning the applications in the dynamics of cell populations, with a spatial distribution of cells, we may associate an energy density $e(u)$, that is an internal energy per unit volume of an evolving spatial pattern, where $u(x, t)$ denotes the cell density (see $[6,14])$. In this case, the total energy $E(u)$ in a volume $V$ is given by

$$
E(u)=\int_{V} e(u) d x
$$

The change in energy $\delta E$, that is the work done in changing states by an amount $\delta u$, is given by the variational derivative $\delta E / \delta u$ which defines a potential

$$
\mu(u)=\frac{\delta E}{\delta u}=e^{\prime}(u) .
$$

The gradient of the potential $\mu$ produces a flux $J$, which is classically proportional to this gradient, that is

$$
J=-k \mu^{\prime}(u)
$$

By using (1.6) and (1.7), the continuity equation for the density $u$ is

$$
\frac{\partial u}{\partial t}=\left(a(u) u_{x}\right)_{x}, \quad a(u)=k e^{\prime \prime}(u) .
$$

Writing out the diffusion term in full, we end up with the nonlinear operator that appears in (1.1a), in the special case where it holds $a^{\prime}(u)=b(u)$, that is the porous medium case. Also, the nonlinearity $\sigma(u)$ may stand for the possible growth dynamics.

For completeness, let us mention some of the results, concerning the large time behavior of bounded solutions of nonlinear diffusion equations. Most of them are related to porous medium type equations (degenerate, monotone diffusion). In [4], the existence of a global attractor for the one-dimensional porous medium equation, attracting all orbits starting from $L^{\infty}$-initial data, is demonstrated. Extensive studies in $[1,13,15]$ show that the $\omega$-limit set is contained 
in the set of stationary solutions. Extensions for the unbounded domain case can be found in $[10,11]$. We also mention $[2,7,8]$ on the existence of global attractors for degenerate or nondegenerate quasilinear parabolic equations.

The principal assumption that will be used throughout this paper in the study of problem (1.1) is the following assumption.

Hypothesis 1.1. $a, b, \sigma \in C^{2}(\mathbb{R}), \lambda \in \mathbb{R}$, and there exists $c_{*}>0$ such that $a(s) \geq c_{*}$ (i.e., we consider nondegenerate but nonmonotone diffusion).

Due to the nonmonotonicity, the standard compactness methods on existence of solutions are not sufficient. To this end, the diffusion operator is treated as a semibounded operator within the functional setting of an admissible triple. This procedure allows for the construction of unique solutions in $C_{w}\left([0, T], H^{2} \cap\right.$ $H_{0}^{1}(\Omega)$ ), the space of weakly continuous functions $u:[0, T] \rightarrow H^{2} \cap H_{0}^{1}(\Omega)$.

The existence of a global attractor in the phase space $H=H^{2} \cap H_{0}^{1}(\Omega)$ is proved in Section 3. The result is shown assuming monotonicity for the nonlinearity $b(\cdot)$, considered to be nonincreasing. Nevertheless, this assumption does not imply monotonicity for the diffusion operator itself. An important feature is that this assumption is sufficient to prove further regularity with respect to time for the solutions of (1.1) constructed in Section 2. Further, using this result, we may define the semigroup $S(t): u_{0} \in H \mapsto u(t) \in H$, corresponding to our problem.

We conclude by recalling some well-known results, which will be frequently used (see, $[16,17,18,19])$.

Lemma 1.2 (Gagliardo-Nirenberg inequality). Let $1 \leq p, q, r \leq \infty, j$ an integer, $0 \leq j \leq m$, and $j / m \leq \theta \leq 1$. Then

$$
\left\|D^{j} u\right\|_{p} \leq \text { const }\|u\|_{q}^{1-\theta}\left\|D^{m} u\right\|_{r}^{\theta}, \quad u \in L^{q} \cap W^{m, r}(\Omega), \Omega \subseteq \mathbb{R}^{n},
$$

where

$$
\frac{1}{p}=\frac{j}{n}+\theta\left(\frac{1}{r}-\frac{m}{n}\right)+\frac{1-\theta}{q} .
$$

If $m-j-n / r$ is not a nonnegative integer, then the inequality holds for $j / m \leq \theta<1$.

Lemma 1.3 (uniform Gronwall). Let $g, h, y$ be three positive locally integrable functions for $t_{0} \leq t<\infty$ which satisfy

$$
\begin{gathered}
\frac{d y}{d t} \leq g y+h, \quad \forall t \geq t_{0}, \\
\int_{t}^{t+r} g(s) d s \leq \alpha_{1}, \quad \int_{t}^{t+r} h(s) d s \leq \alpha_{2}, \quad \int_{t}^{t+r} y(s) d s \leq \alpha_{3},
\end{gathered}
$$


for all $t \geq t_{0}$, where $\alpha_{1}, \alpha_{2}$, and $\alpha_{3}$ are positive constants. Then

$$
y(t+r) \leq\left(\frac{\alpha_{3}}{r}+\alpha_{2}\right) \exp \left(\alpha_{1}\right), \quad \forall t \geq t_{0}
$$

We also use the short (equivalent) norms $\left\|u_{x}\right\|_{2},\left\|u_{x x}\right\|_{2}$, and $\left\|u_{x x x}\right\|_{2}$ in $H_{0}^{1}(\Omega)$, $H^{2} \cap H_{0}^{1}(\Omega)$, and $H^{3} \cap H_{0}^{1}(\Omega)$, respectively (see Section 3 ). From the embedding $H^{k} \cap H_{0}^{1}(\Omega) \hookrightarrow C_{b}^{k-1}(\Omega), k=1,2, \ldots$, and the Poincaré inequality (see [9, page 242]), we have

$$
\left\|u^{(k-1)}\right\|_{\infty} \leq \text { const }\|u\|_{H^{k} \cap H_{0}^{1}} \leq \text { const }\left\|u^{(k)}\right\|_{2}
$$

\section{Local existence}

To obtain results on local existence of solutions, we intend to write problem (1.1) as a nonlinear evolution equation in an appropriate functional setting. More precisely, we will consider an admissible triple of Banach spaces, which is defined as follows (see $[17,18]$ and $[19$, page 784$]$ ).

Definition 2.1. An admissible triple $V \hookrightarrow H \hookrightarrow W$ has the following properties: (i) $H$ is a real separable Hilbert space with scalar product $(\cdot \mid \cdot)_{H}$, (ii) $\{V, W\}$ is a dual pair of real separable Banach spaces with the corresponding bilinear form $\langle\cdot, \cdot\rangle$ (i.e., $\langle\cdot, \cdot\rangle$ is continuous, $\langle w, v\rangle=0$, for every $w \in W$, implies $v=0$, and $\langle w, v\rangle=0$, for every $v \in V$, implies $w=0$ ), (iii) the embeddings $V \hookrightarrow H \hookrightarrow W$ are continuous and dense, (iv) it holds $\langle h, v\rangle=(h \mid v)_{H}$, for all $h \in H, v \in V$.

Clearly, an admissible triple generalizes the notion of the evolution triple, in the sense that for an admissible triple it may hold $W \neq V^{*}$. This generalization is necessary in order to tackle the extended version of diffusion in hand. For problem (1.1), we select the spaces

$$
V=H^{4} \cap H_{0}^{1}(\Omega), \quad H=H^{2} \cap H_{0}^{1}(\Omega), \quad W=L^{2}(\Omega) .
$$

Lemma 2.2. The embedding $V \hookrightarrow H \hookrightarrow W$ for the spaces (2.1) defines an admissible triple.

Sketch of the proof. Consider the bilinear form $\langle\cdot, \cdot\rangle: W \times V \mapsto \mathbb{R}$, defined by the integral

$$
\langle w, v\rangle=\int_{\Omega} v w+w v_{x x x x} d x, \quad \forall v \in V, w \in W
$$

Now, it is easy to check that the inner product stemming from the bilinear form $\langle\cdot, \cdot\rangle$

$$
(w \mid v)_{H}=\int_{\Omega} v w+w_{x x} v_{x x} d x, \quad \text { for every } w \in H, v \in H
$$


induces an equivalent norm in $H$. We also have that

$$
\begin{aligned}
|\langle w, v\rangle| & =\left|\int_{\Omega} v w+w v_{x x x x} d x\right| \\
& \leq\|w\|_{2}\|v\|_{2}+\|w\|_{2}\left\|v_{x x x x}\right\|_{2} \\
& \leq c\|w\|_{W}\|v\|_{V},
\end{aligned}
$$

hence the bilinear form $\langle\cdot, \cdot\rangle$ is continuous. Now assume that, for some $w \in$ $W$, it holds $\langle w, v\rangle=0$, for every $v \in V$. Classical arguments on existence and regularity of solutions for linear elliptic equations (see [12, Chapter II]) imply the existence of solutions for the problem

$$
v-v_{x x x x}=w, \quad v \in V .
$$

For this solution $v$, we have that

$$
0=\langle w, v\rangle=\int_{\Omega} w^{2} d x
$$

which implies that $w=0$ and the proof is complete.

We introduce the nonlinear operators $\mathbf{A}, \mathbf{B}: V \mapsto W$ defined by

$$
\mathbf{A} u=-a(u) u_{x x}, \quad \mathbf{B} u=-b(u) u_{x}^{2}
$$

The following results outline the basic properties of the operators $\mathbf{A}$ and $\mathbf{B}$.

Proposition 2.3. The operator $\mathbf{A}+\mathbf{B}: H \mapsto W$ is bounded on bounded sets of $H$. Proof. Let $B=B_{H}(R)$ be a closed ball in $H$. We will show that there exist constants $K_{1}(R)$ and $K_{2}(R)$ such that

$$
\|\mathbf{A} u\|_{2} \leq K_{1}(R)\|u\|_{H}, \quad\|\mathbf{B} u\|_{2} \leq K_{2}(R)\|u\|_{H}, \quad \forall u \in B .
$$

Since $a, b, \sigma \in C^{2}(\mathbb{R})$ and the embedding $H \hookrightarrow C_{b}^{1}(\Omega)$ is continuous, it follows that there exist constants $C_{1, m}(R)$ and $C_{2, m}(R), m=0,1,2$, such that

$$
\begin{array}{ll}
\sup _{x \in \Omega}\left|a^{(m)}(u(x))\right| \leq C_{1, m}(R), & m=0,1,2, \\
\sup _{x \in \Omega}\left|b^{(m)}(u(x))\right| \leq C_{2, m}(R), & m=0,1,2 .
\end{array}
$$

Using (2.9), (2.10), and the fact that $H_{0}^{1}(\Omega)$ is a generalized Banach algebra, we may obtain the inequalities

$$
\begin{aligned}
\|\mathbf{A} u\|_{2} & \leq \sup _{x \in \Omega} \mid a(u(x))\|\| u_{x x}\left\|_{2} \leq K_{1}(R)\right\| u \|_{H}, \\
\|\mathbf{B} u\|_{2} & \leq \sup _{x \in \Omega}\left|b(u(x))\|\| u_{x}^{2}\left\|_{2} \leq \mathrm{const} \sup _{x \in \Omega}|b(u(x))|\right\| u \|_{H}^{2}\right. \\
& \leq K_{2}(R)\|u\|_{H} .
\end{aligned}
$$


526 Semibounded evolution equation

Finally, we conclude that

$$
\|(\mathbf{A}+\mathbf{B}) u\|_{2} \leq K(R)\|u\|_{H}
$$

where $K(R)=\max \left\{K_{1}(R), K_{2}(R)\right\}$.

Proposition 2.4. The operator $\mathbf{A}+\mathbf{B}: H \mapsto W$ is locally Lipschitz continuous.

Proof. Let $u, v \in B=B_{H}(R)$ be a closed ball in $H$. We have that

$$
\|\mathbf{A} u-\mathbf{A} v\|_{2} \leq\left\|(a(u)-a(v)) v_{x x}\right\|_{2}+\left\|a(u)\left(u_{x x}-v_{x x}\right)\right\|_{2} .
$$

From the mean value theorem and (2.9), we get

$$
\begin{aligned}
|a(u(x))-a(v(x))| & \leq C_{1,1}(R)|u(x)-v(x)|, \\
\left|a^{\prime}(u(x))-a^{\prime}(v(x))\right| & \leq C_{1,2}(R)|u(x)-v(x)| .
\end{aligned}
$$

Therefore,

$$
\begin{gathered}
\left\|(a(u)-a(v)) v_{x x}\right\|_{2}^{2} \leq C_{1,1}(R)^{2}\|u-v\|_{\infty}^{2}\left\|v_{x x}\right\|_{2}^{2} \leq C(R)\|u-v\|_{H}^{2}, \\
\left\|a(u)\left(u_{x x}-v_{x x}\right)\right\|_{2}^{2} \leq C_{1,0}^{2}(R)\left\|u_{x x}-v_{x x}\right\|_{2}^{2} \leq C(R)\|u-v\|_{H}^{2},
\end{gathered}
$$

where $C(R)$ is a common symbol for the constants. Similar inequalities hold for the operator B. So finally it holds that

$$
\|(\mathbf{A}+\mathbf{B}) u-(\mathbf{A}+\mathbf{B}) v\|_{2} \leq C(R)\|u-v\|_{H} .
$$

Proposition 2.5. The operator $\mathbf{A}+\mathbf{B}: H \mapsto W$ is semibounded.

Proof. By definition, it must be proved that there exists a monotone increasing function $d_{1} \in C^{1}(\mathbb{R})$ such that

$$
\langle(\mathbf{A}+\mathbf{B}) u, u\rangle \geq-d_{1}\left(\|u\|_{H}^{2}\right), \quad \text { for every } u \in V .
$$

Let $u \in C_{0}^{\infty}(\Omega) \cap C(\bar{\Omega})$. For the operator $\mathbf{A}$, it holds

$$
\langle\mathbf{A} u, u\rangle=\int_{\Omega} \mathbf{A} u u d x+\int_{\Omega} \mathbf{A} u u_{x x x x} d x
$$


Integration by parts in the second integral on the right-hand side of (2.19) gives

$$
\begin{aligned}
-\int_{\Omega} a(u) u_{x x} u_{x x x x} d x= & -\frac{1}{2} \int_{\Omega} a^{\prime \prime}(u) u_{x}^{2} u_{x x}^{2} d x-\frac{1}{2} \int_{\Omega} a^{\prime}(u) u_{x x}^{3} d x \\
& +\int_{\Omega} a(u) u_{x x x}^{2} d x
\end{aligned}
$$

Using Lemma 1.2, we obtain the inequality

$$
\left\|u_{x x}\right\|_{4} \leq \text { const }\|u\|_{2}^{1 / 4}\left\|u_{x x x}\right\|_{2}^{3 / 4}
$$

which, with the aid of (2.9) and Young's inequality, gives the following estimate:

$$
\begin{aligned}
-\frac{1}{2} \int_{\Omega} a^{\prime \prime}(u) u_{x}^{2} u_{x x}^{2} d x-\frac{1}{2} \int_{\Omega} a^{\prime}(u) u_{x x}^{3} d x \\
\\
\geq-C_{1,2}\left\|u_{x}\right\|_{\infty}^{2}\left\|u_{x x}\right\|_{2}^{2}-C_{1,1}\left\|u_{x x}\right\|_{2}\left\|u_{x x}\right\|_{4}^{2} \\
\geq-\hat{C}_{1}\|u\|_{H}^{4}-\hat{C}_{2}\|u\|_{H}\|u\|_{2}^{1 / 2}\left\|u_{x x x}\right\|_{2}^{3 / 2} \\
\geq-\hat{C}_{1}\|u\|_{H}^{4}-\hat{C}_{3}\|u\|_{H}^{3 / 2}\left\|u_{x x x}\right\|_{2}^{3 / 2} \\
\geq-\hat{C}_{1}\|u\|_{H}^{4}-\hat{C}_{4}\|u\|_{H}^{6}-\frac{c_{*}}{2}\left\|u_{x x x}\right\|_{2}^{2} .
\end{aligned}
$$

For the first integral of the right-hand side of (2.19), we have

$$
-\int_{\Omega} a(u) u_{x x} u d x \geq-C_{1,0}\|u\|_{\infty}\left\|u_{x x}\right\|_{1} \geq-\hat{C}_{0}\|u\|_{H}^{2}
$$

Using Hypothesis 1.1, (2.19), (2.20), (2.22), (2.23), and density arguments, we obtain that

$$
\langle\mathbf{A} u, u\rangle \geq-\hat{C}_{0}\|u\|_{H}^{2}-\hat{C}_{1}\|u\|_{H}^{4}-\hat{C}_{4}\|u\|_{H}^{6}:=-d_{1,1}\left(\|u\|_{H}^{2}\right)
$$

A similar procedure may be followed for the operator $\mathbf{B}$, to derive the relation

$$
\langle\mathbf{B} u, u\rangle \geq-d_{1,2}\left(\|u\|_{H}^{2}\right)
$$

Finally, from estimates (2.24) and (2.25) we get that there exists a monotone increasing $C^{1}$ - function $d_{1}: \mathbb{R} \rightarrow \mathbb{R}$ satisfying (2.18).

The previous propositions enable us to show local existence of solutions. The result is stated as follows. 
Theorem 2.6. Let $u_{0}, f \in H$. Assume that Hypothesis 1.1 is satisfied. Then there exists $T>0$ such that problem (1.1) has a unique solution

$$
u \in C_{w}([0, T], H), \quad u_{t} \in C_{w}([0, T], W) .
$$

Moreover, the solution $u:[0, T] \rightarrow W$ is Lipschitz continuous.

Proof. (A) Existence: the first step is to show existence of at least one solution in a finite dimensional subspace $V_{n}=\operatorname{span}\left\{e_{1}, \ldots, e_{n}\right\}$ of $V$, where $\left\{e_{i}\right\}_{i \geq 1}$ is an orthonormal basis of $V_{n}$ with respect to $(\cdot \mid \cdot)_{H}$. It holds that $\overline{U_{n} V_{n}}=V \hookrightarrow H$.

We define the linear and continuous operator $\tilde{P}_{n}: W \mapsto V$ as

$$
\tilde{P}_{n} w=\sum_{i}^{n}\left\langle w, e_{i}\right\rangle e_{i}, \quad w \in W
$$

Now, the Galerkin equation for problem (1.1) on $V_{n} \hookrightarrow V \hookrightarrow H$ reads

$$
u_{n}^{\prime}(t)+\tilde{P}_{n}(\mathbf{A}+\mathbf{B}) u_{n}(t)=\tilde{P}_{n} \mathbf{C} u_{n}(t), \quad t \in[0, T], u_{n}(0)=\tilde{P}_{n} u_{0},
$$

where

$$
\mathrm{C} u_{n}(t)=\lambda \sigma\left(u_{n}(t)\right)+f
$$

Using Propositions 2.3 and 2.4, Peano's theorem justifies the existence of a $C^{1}$ solution for (2.28), $u_{n}:\left[0, T_{0}\right] \rightarrow V_{n}$, for some $T_{0}>0$ which depends on $n$.

The next step is to obtain an a priori estimate for $u_{n}$ in $H$. Note that $\tilde{P}_{n}$ : $H \mapsto V_{n}$ is an orthogonal projection onto the space $V_{n}$, since it holds $\tilde{P}_{n} u=$ $\sum_{i}^{n}\left(u \mid e_{i}\right)_{H} e_{i}, u \in H$. Since $u_{n}$ is continuous on [0, $\left.T_{0}\right]$, (2.28) implies that

$$
\begin{aligned}
\left(u_{n}^{\prime} \mid u_{n}\right)_{H} & =-\left(\tilde{P}_{n}(\mathbf{A}+\mathbf{B}) u_{n} \mid u_{n}\right)_{H}+\left(\tilde{P}_{n} \mathbf{C} u_{n} \mid u_{n}\right)_{H} \\
& =-\left\langle(\mathbf{A}+\mathbf{B}) u_{n}, u_{n}\right\rangle+\left\langle\mathbf{C} u_{n}, u_{n}\right\rangle .
\end{aligned}
$$

Now, it is not hard to verify that there exists a monotone increasing function $d_{2} \in C^{1}(\mathbb{R})$ such that

$$
|\langle\mathrm{C} u, u\rangle| \leq d_{2}\left(\|u\|_{H}^{2}\right), \quad \forall u \in V .
$$

Hence, from (2.18), (2.30), and (2.31) we obtain the differential inequality

$$
\frac{d}{d t}\left\|u_{n}(t)\right\|_{H}^{2} \leq 2 d\left(\left\|u_{n}(t)\right\|_{H}^{2}\right), \quad t \in\left[0, T_{0}\right]
$$


where $\left\|u_{n}(0)\right\|_{H}=\left\|P_{n} u_{0}\right\|_{H} \leq\left\|u_{0}\right\|_{H}$. Since the function $d(\cdot)$ is Lipschitz continuous as a $C^{1}$ function, we may apply the theorem of Picard-Lindelöf to conclude that there exists a $T>0$, this time independent of $n$, such that

$$
\left\|u_{n}(t)\right\|_{H}^{2} \leq \max _{t \in[0, T]} g(t) \leq R, \quad t \in[0, T]
$$

Finally, using standard continuation arguments, we can extend the solution $u_{n}$ to the interval $[0, T]$.

Now, from (2.33) we have that there exists a subsequence, denoted again by $\left\{u_{n}\right\}$, such that

$$
u_{n}(t) \longrightarrow u(t), \quad \text { in } H, \text { as } n \longrightarrow \infty,
$$

at least in a dense countable subset of $[0, T]$. Let $v \in V_{k} \hookrightarrow H, k \leq n$. Since $\tilde{P}_{n} v=$ $v$, for every $k \leq n$, it follows that

$$
\left(u_{n}^{\prime}(t) \mid v\right)_{H}=-\left(\tilde{P}_{n}(\mathbf{A}+\mathbf{B}-\mathbf{C}) u_{n}(t) \mid v\right)_{H}=-\left\langle(\mathbf{A}+\mathbf{B}-\mathbf{C}) u_{n}(t), v\right\rangle .
$$

Using Proposition 2.3 and estimate (2.33), we conclude that $\left(u_{n}(t) \mid v\right)_{H}$ is equicontinuous on $[0, T]$, which implies that $(2.34)$ holds in the whole interval $[0, T]$. Finally, passing to the limit to (2.35) and using density of $\bigcup_{k} V_{k}$ in $H$, we obtain that $u \in C_{w}([0, T], H), u_{t} \in C_{w}([0, T], W)$ is a solution for problem (1.1) and as a consequence, $u:[0, T] \rightarrow W$ is Lipschitz continuous.

(B) Uniqueness: the difference of solutions $w=u-v$ of problem (1.1) satisfies the following initial value problem:

$$
w_{t}-a(u) w_{x x}-\mathrm{A}(u, v) v_{x x}-B(u, v)-\lambda \Sigma(u, v)=0, \quad w(0)=0,
$$

where $\mathrm{A}(u, v)=a(u)-a(v), B(u, v)=(b(u)-b(v)) v_{x}^{2}+b(u)\left(u_{x}^{2}-v_{x}^{2}\right)$, and $\Sigma(u$, $v)=\sigma(u)-\sigma(v)$. Multiplying (2.36) by $u$ and integrating over $\Omega$, we obtain the equation

$$
\begin{aligned}
\frac{1}{2} \frac{d}{d t}\|w\|_{2}^{2} & +\int_{\Omega} a^{\prime}(u) v_{x} w w_{x} d x+\int_{\Omega}(a(u)-a(v)) w_{x} v_{x} d x \\
& +\int_{\Omega} a^{\prime}(u) u_{x} w w_{x} d x+\int_{\Omega}\left(a^{\prime}(u)-a^{\prime}(v)\right) v_{x}^{2} w d x \\
& -\int_{\Omega}(b(u)-b(v)) v_{x}^{2} w d x-\int_{\Omega} b(u)\left(u_{x}^{2}-v_{x}^{2}\right) w d x \\
& +\int_{\Omega} a(u) w_{x}^{2} d x-\lambda \int_{\Omega}(\sigma(u)-\sigma(v)) w d x=0 .
\end{aligned}
$$


Using estimate (2.33) and relations (2.9), (2.10), and (2.15) the following estimates are derived:

$$
\begin{aligned}
\left|\int_{\Omega}\left(a^{\prime}(u)-a^{\prime}(v)\right) v_{x}^{2} w d x\right| & \leq C_{1,2}\left\|v_{x}\right\|_{\infty}^{2}\|w\|_{2}^{2} \leq C(R)\|w\|_{2}^{2}, \\
\left|\int_{\Omega} a^{\prime}(u) v_{x} w w_{x} d x\right| & \leq C_{1,1}\left\|v_{x}\right\|_{\infty}\|w\|_{2}\left\|w_{x}\right\|_{2} \\
& \leq \epsilon_{0}\left\|w_{x}\right\|_{2}^{2}+C(R)\|w\|_{2}^{2} .
\end{aligned}
$$

The rest of the integrals in (2.37) can be estimated in a similar way. Hence, for sufficiently small $\epsilon_{0}$, we get the inequality

$$
\frac{1}{2} \frac{d}{d t}\|w(t)\|_{W}^{2}+\frac{c_{*}}{2}\left\|w_{x}\right\|_{2}^{2} \leq C\|w(t)\|_{W}^{2} .
$$

Application of the standard Gronwall's lemma implies uniqueness.

\section{Existence of a global attractor in $H$}

In this section, we discuss the asymptotic behavior of solutions of the nonlinear parabolic problem (1.1). To this end, in addition to the principal hypothesis, Hypothesis 1.1, we assume that the nonlinear functions $b, \sigma$ satisfy the following hypothesis.

Hypothesis 3.1. $b^{\prime}(s) \leq 0$ and there exist $c_{m}>0$, such that $\left|\sigma^{(m)}(s)\right| \leq c_{m}|s|$, for all $m=0,1,2$.

First, we prove that under the extra hypothesis, Hypothesis 3.1, the unique local solution $u(x, t)$ of problem (1.1), obtained in Theorem 2.6, exists globally in time. We denote by $\lambda_{*}$ the positive constant induced by Poincaré's inequality. Lemma 3.2. Let Hypotheses 1.1 and 3.1 be fulfilled and $u_{0}, f \in H$. Assume also that

$$
\lambda<\frac{c_{*} \lambda_{*}}{2 c_{0}}
$$

Then there exists a constant $\rho_{2}$ independent of $t$, such that,

$$
\limsup _{t \rightarrow \infty}\left\|u_{x}(t)\right\|_{2} \leq \rho_{2} .
$$

Proof. We multiply (1.1a) by $-u_{x x}$ and integrate over $\Omega$ to get

$$
\begin{aligned}
\frac{1}{2} \frac{d}{d t}\left\|u_{x}\right\|_{2}^{2} & +\int_{\Omega} a(u) u_{x x}^{2} d x+\int_{\Omega} b(u) u_{x}^{2} u_{x x} d x \\
& +\lambda \int_{\Omega} \sigma(u) u_{x x} d x=\int_{\Omega} f u_{x x} d x
\end{aligned}
$$


Using Hypothesis 1.1, we observe that

$$
\int_{\Omega} a(u) u_{x x}^{2} d x \geq c_{*}\left\|u_{x x}\right\|_{2}^{2}
$$

whereas from Hypothesis 3.1 we have

$$
\int_{\Omega} b(u) u_{x}^{2} u_{x x} d x=-\frac{1}{3} \int_{\Omega} b^{\prime}(u) u_{x}^{4} d x \geq 0
$$

Furthermore, Hypothesis 3.1, together with Poincaré's inequality

$$
\|u\|_{2} \leq \lambda_{*}^{-1 / 2}\left\|u_{x}\right\|_{2}
$$

implies that

$$
\lambda\left|\int_{\Omega} \sigma(u) u_{x x} d x\right| \leq \lambda c_{0}\|u\|_{2}\left\|u_{x x}\right\|_{2} \leq \lambda \lambda_{*}^{-1} c_{0}\left\|u_{x x}\right\|_{2}^{2}
$$

Relations (3.3), (3.4), and (3.7) imply that

$$
\frac{d}{d t}\left\|u_{x}(t)\right\|_{2}^{2}+\alpha\left\|u_{x x}(t)\right\|_{2}^{2} \leq \frac{1}{c_{*}}\|f\|_{2}^{2}
$$

where $\alpha=c_{*}-2 c_{0} \lambda \lambda_{*}^{-1}$. Applying again Poincaré's inequality (3.6) to the above estimate (3.8), we get

$$
\frac{d}{d t}\left\|u_{x}(t)\right\|_{2}^{2}+\alpha \lambda_{*}\left\|u_{x}(t)\right\|_{2}^{2} \leq \frac{1}{c_{*}}\|f\|_{2}^{2}
$$

If assumption (3.1) is satisfied, that is, $\alpha>0$ Gronwall's lemma leads to the following estimate:

$$
\left\|u_{x}(t)\right\|_{2}^{2} \leq\left\|u_{x}(0)\right\|_{2}^{2} \exp \left(-\alpha \lambda_{*} t\right)+\frac{1}{\alpha c_{*} \lambda_{*}}\|f\|_{2}^{2}\left(1-\exp \left(-\alpha \lambda_{*} t\right)\right)
$$

Letting $t \rightarrow \infty$, from estimate (3.10) we obtain that

$$
\limsup _{t \rightarrow \infty}\left\|u_{x}(t)\right\|_{2}^{2} \leq \rho_{2}^{2}
$$

where $\rho_{2}^{2}=\left(1 / \alpha c_{*} \lambda_{*}\right)\|f\|_{2}^{2}$ and the proof is completed. 
532 Semibounded evolution equation

Let $\mathscr{B}$ be a bounded set of $H$, included in a ball $B_{H}(0, M)$ of $H$, centered at 0 of radius $M$. Assuming that $u_{0} \in \mathscr{B}$, we infer from Lemma 3.2 that for $\rho_{2}^{\prime}>\rho_{2}$, there exists $t_{0}\left(\mathscr{B}, \rho_{2}^{\prime}\right)>0$ such that for $t \geq t_{0}\left(\mathscr{B}, \rho_{2}^{\prime}\right)$

$$
\left\|u_{x}(t)\right\|_{2} \leq \rho_{2}^{\prime}, \quad\|u(t)\|_{2} \leq \rho_{1}=\lambda_{*}^{-1 / 2} \rho_{2}^{\prime} .
$$

Integrating (3.8) with respect to $t$, it follows that for every $r>0$

$$
\alpha \int_{t}^{t+r}\left\|u_{x x}(s)\right\|_{2}^{2} d s \leq \frac{r}{c_{*}}\|f\|_{2}^{2}+\left\|u_{x}(t)\right\|_{2}^{2} .
$$

Once again, letting $t \rightarrow \infty$, we obtain from inequality (3.12) that

$$
\underset{t \rightarrow \infty}{\limsup } \int_{t}^{t+r}\left\|u_{x x}(s)\right\|_{2}^{2} d s \leq \frac{r}{\alpha c_{*}}\|f\|_{2}^{2}+\frac{\rho_{2}^{2}}{\alpha}, \quad \text { for every } r>0 .
$$

and for $t \geq t_{0}\left(\mathscr{B}, \rho_{2}^{\prime}\right)$

$$
\int_{t}^{t+r}\left\|u_{x x}(s)\right\|_{2}^{2} d s \leq \frac{r}{\alpha c_{*}}\|f\|_{2}^{2}+\frac{\rho_{2}^{\prime 2}}{\alpha}, \quad \text { for every } r>0 .
$$

Lemma 3.3. Let Hypotheses 1.1 and 3.1 be fulfilled, $u_{0} \in \mathscr{B}$, and $f \in H$. Assume also that (3.1) is satisfied. Then there exists a constant $\rho_{3}$ independent of $t$, and $t_{1}>0$ such that

$$
\left\|u_{x x}(t)\right\|_{2} \leq \rho_{3}, \quad \text { for } t \geq t_{1} .
$$

Proof. Multiply (1.1a) by $u_{x x x x}$ and integrate over $\Omega$ to get

$$
\begin{aligned}
\frac{1}{2} \frac{d}{d t}\left\|u_{x x}\right\|_{2}^{2} & +\int_{\Omega} a^{\prime}(u) u_{x} u_{x x} u_{x x x} d x+\int_{\Omega} a(u) u_{x x x}^{2} d x \\
& +2 \int_{\Omega} b(u) u_{x} u_{x x} u_{x x x} d x+\lambda \int_{\Omega} \sigma^{\prime}(u) u_{x} u_{x x x} d x \\
& +\int_{\Omega} b^{\prime}(u) u_{x}^{3} u_{x x x} d x \\
= & -\int_{\Omega} f_{x} u_{x x x} d x .
\end{aligned}
$$

Using inequalities (1.13), (3.12), and Hypothesis 1.1, we obtain that inequalities (2.9) and (2.10) hold, for all $t \geq t_{0}\left(\mathscr{B}, \rho_{2}^{\prime}\right)$, with $R$ replaced by $\rho_{2}^{\prime}$. It follows that

$$
\begin{aligned}
\left|\int_{\Omega} a^{\prime}(u) u_{x} u_{x x} u_{x x x} d x\right| & \leq C_{1,1}\left\|u_{x}\right\|_{\infty}\left\|u_{x x}\right\|_{2}\left\|u_{x x x}\right\|_{2} \\
& \leq C_{1,1} \text { const }\left\|u_{x x}\right\|_{2}^{2}\left\|u_{x x x}\right\|_{2} \\
& \leq C_{1}\left\|u_{x x}\right\|_{2}^{4}+\epsilon_{1}\left\|u_{x x x}\right\|_{2}^{2} .
\end{aligned}
$$


Applying Lemma 1.2, we obtain the inequality

$$
\left\|u_{x}\right\|_{6} \leq \text { const }\|u\|_{2}^{1 / 3}\left\|u_{x x}\right\|_{2}^{2 / 3}
$$

which can be used to get the estimate

$$
\begin{aligned}
\left|\int_{\Omega} b^{\prime}(u) u_{x}^{3} u_{x x x} d x\right| & \leq C_{2,1}\left\|u_{x}\right\|_{6}^{3}\left\|u_{x x x}\right\|_{2} \\
& \leq C_{2,1} \text { const }\|u\|_{2}\left\|u_{x x}\right\|_{2}^{2}\left\|u_{x x x}\right\|_{2} \\
& \leq C_{2}\left\|u_{x x}\right\|^{4}+\epsilon_{1}\left\|u_{x x x}\right\|_{2}^{2} .
\end{aligned}
$$

We also have that the estimate

$$
\begin{aligned}
\lambda\left|\int_{\Omega} \sigma^{\prime}(u) u_{x} u_{x x x} d x\right| & \leq \lambda c_{1}\|u\|_{\infty}\left\|u_{x}\right\|_{2}\left\|u_{x x x}\right\|_{2} \\
& \leq \lambda c_{1} \text { const }\left\|u_{x x}\right\|_{2}^{2}\left\|u_{x x x}\right\|_{2} \\
& \leq C_{3}\left\|u_{x x}\right\|_{2}^{4}+\epsilon_{1}\left\|u_{x x x}\right\|_{2}^{2} .
\end{aligned}
$$

The rest of the integral terms in (3.17) can be bounded similarly. Thus, for sufficiently small $\epsilon_{1}$, we get the inequalities

$$
\begin{gathered}
\frac{d}{d t}\left\|u_{x x}(t)\right\|_{2}^{2}+c_{*}\left\|u_{x x x}(t)\right\|_{2}^{2} \leq M_{1}+M_{2}\left\|u_{x x}(t)\right\|_{2}^{4}, \\
\frac{d}{d t}\left\|u_{x x}(t)\right\|_{2}^{2} \leq M_{1}+M_{2}\left\|u_{x x}(t)\right\|_{2}^{4},
\end{gathered}
$$

where $M_{1}$ and $M_{2}$ are independent of $t$. We set $y(t)=\left\|u_{x x}(t)\right\|_{2}^{2}, h(t)=M_{1}$, and $g(t)=M_{2}\left\|u_{x x}(t)\right\|_{2}^{2}$. For fixed $r>0$, we use (3.15) to deduce that

$$
\int_{t}^{t+r} g(s) d s \leq \alpha_{1}, \quad \int_{t}^{t+r} h(s) d s \leq \alpha_{2}, \quad \int_{t}^{t+r} y(s) d s \leq \alpha_{3},
$$

for all $t \geq t_{0}\left(\mathscr{B}, \rho_{2}^{\prime}\right)$, where $\alpha_{1}=M_{2} \alpha_{3}, \alpha_{2}=M_{1} r$, and $\alpha_{3}=\left(r / \alpha c_{*}\right)\|f\|_{2}^{2}+\rho_{2}^{\prime 2} / \alpha$. Applying uniform Gronwall's lemma (Lemma 1.3) to the differential inequality (3.23), we conclude that

$$
\left\|u_{x x}(t)\right\|_{2}^{2} \leq\left(\frac{\alpha_{3}}{r}+\alpha_{2}\right) \exp \left(\alpha_{1}\right):=\rho_{3}^{2}, \quad \forall t \geq t_{0}\left(\mathscr{B}, \rho_{2}^{\prime}\right)+r
$$

and the proof is complete.

Lemma 3.4. Let Hypotheses 1.1 and 3.1 be fulfilled, $u_{0} \in \mathscr{B}$, and $f \in H$. Assume also that (3.1) is satisfied. Then, there exists a constant $\rho_{4}$ independent of $t$ and $t_{2}>0$, such that

$$
\left\|u_{x x x}(t)\right\|_{2} \leq \rho_{4}, \quad \text { for } t \geq t_{2}
$$


534 Semibounded evolution equation

Proof. We multiply (1.1a) by $-u^{(6)}$ and integrate over $\Omega$ to get the equation

$$
\begin{aligned}
\frac{1}{2} \frac{d}{d t}\left\|u_{x x x}\right\|_{2}^{2} & +\int_{\Omega} a(u) u_{x x x x}^{2} d x+\int_{\Omega} \mathbf{A}_{1}(u) u_{x}^{2} u_{x x} u_{x x x x} d x \\
& +2 \int_{\Omega} \mathbf{A}_{2}(u) u_{x} u_{x x x} u_{x x x x} d x+\int_{\Omega} \mathbf{A}_{3}(u) u_{x x}^{2} u_{x x x x} d x \\
& +\lambda \int_{\Omega}\left(\sigma^{\prime \prime}(u) u_{x}^{2}+\sigma^{\prime}(u) u_{x x}\right) u_{x x x x} d x+\int_{\Omega} b^{\prime \prime}(u) u_{x}^{4} u_{x x x x} d x \\
= & -\int_{\Omega} f_{x x} u_{x x x x} d x,
\end{aligned}
$$

where $\mathbf{A}_{1}(u)=a^{\prime \prime}(u)+5 b^{\prime}(u), \mathbf{A}_{2}(u)=a^{\prime}(u)+b(u)$, and $\mathbf{A}_{3}(u)=a^{\prime}(u)+2 b(u)$. Similarly to Lemma 3.3 , we arrive at the inequality

$$
\frac{d}{d t}\left\|u_{x x x}(t)\right\|_{2}^{2}+c_{*}\left\|u_{x x x x}(t)\right\|_{2}^{2} \leq M_{3}+M_{4}\left\|u_{x x x}(t)\right\|_{2}^{4},
$$

where $M_{3}\left(\rho_{1}, \rho_{2}^{\prime}, \rho_{3}\right)$ and $M_{4}\left(\rho_{1}, \rho_{2}^{\prime}, \rho_{3}\right)$ are independent of $t$. Moreover, from inequality (3.22) we obtain that for fixed $r^{\prime}>0$

$$
\int_{t}^{t+r^{\prime}}\left\|u_{x x x}(s)\right\|^{2} d s \leq \frac{M_{1} r^{\prime}}{c_{*}}+\frac{\rho_{3}^{2}}{c_{*}}\left(M_{2} \rho_{3}^{2} r^{\prime}+1\right) .
$$

Setting $y(t)=\left\|u_{x x x}(t)\right\|_{2}^{2}, h(t)=M_{3}$, and $g(t)=M_{4}\left\|u_{x x x}(t)\right\|_{2}^{2}$, inequality (3.29) implies the following estimates:

$$
\int_{t}^{t+r^{\prime}} g(s) d s \leq \beta_{1}, \quad \int_{t}^{t+r^{\prime}} h(s) d s \leq \beta_{2}, \quad \int_{t}^{t+r^{\prime}} y(s) d s \leq \beta_{3},
$$

where

$$
\beta_{1}=M_{4} \beta_{3}, \quad \beta_{2}=M_{3} r^{\prime}, \quad \beta_{3}=\frac{M_{1} r^{\prime}}{c_{*}}+\frac{\rho_{3}^{2}}{c_{*}}\left(M_{2} \rho_{3}^{2} r^{\prime}+1\right) .
$$

Applying Lemma 1.3 to the differential inequality (3.28), we conclude that

$$
\left\|u_{x x x}(t)\right\|_{2}^{2} \leq\left(\frac{\beta_{3}}{r^{\prime}}+\beta_{2}\right) \exp \left(\beta_{1}\right):=\rho_{4}^{2}, \quad \text { for } t \geq t_{1}+r^{\prime}
$$

to complete the proof.

Next we discuss certain regularity questions of the solution and the solution operator for problem (1.1). 
Proposition 3.5. Let Hypotheses 1.1 and 3.1 be fulfilled and $u_{0}, f \in H$. Then, for the unique solution of (1.1), it holds that $u \in C(0, T ; H)$, for every $T>0$. Moreover, the mapping $S(t): u_{0} \in H \mapsto u(t) \in H$ is continuous.

Proof. We will divide the proof to two parts.

(A) Continuity of Solutions. Consider the dense embeddings

$$
V \hookrightarrow H \hookrightarrow V^{*}
$$

A consequence of relation (3.28) is that $u \in L^{2}(0, T ; V)$, for every $T>0$. Also, it can be easily proved that $u_{t} \in L^{2}(0, T ; W)$. Taking into account the continuous embedding $L^{2}(0, T ; W) \hookrightarrow L^{2}\left(0, T ; V^{*}\right)$, it follows that

$$
u \in \mathcal{W} \equiv\left\{u \in L^{2}(0, T ; V), u_{t} \in L^{2}\left(0, T ; V^{*}\right)\right\} \hookrightarrow C(0, T ; H)
$$

(B) Continuity of the solution mapping. Multiply (2.36) by $w_{x x x x}$ and integrate over $\Omega$ to get the following relation:

$$
\begin{aligned}
\frac{1}{2} \frac{d}{d t}\left\|w_{x x}\right\|_{2}^{2} & +\int_{\Omega} a(u) w_{x x x}^{2} d x+\int_{\Omega} a^{\prime}(u) u_{x} w_{x x} w_{x x x} d x \\
& +\int_{\Omega}\left(a^{\prime}(u)-a^{\prime}(v)\right) v_{x} v_{x x} w_{x x x} d x+\int_{\Omega} a^{\prime}(u) w_{x} v_{x x} w_{x x x} d x \\
& +\int_{\Omega}(a(u)-a(v)) v_{x x x} w_{x x x} d x+\int_{\Omega}\left(b^{\prime}(u)-b^{\prime}(v)\right) v_{x}^{3} w_{x x x} d x \\
& +\int_{\Omega} b^{\prime}(u) v_{x}^{2} w_{x} w_{x x x} d x+\int_{\Omega} b^{\prime}(u)\left(u_{x}+v_{x}\right) u_{x} w_{x} w_{x x x} d x \\
& +\int_{\Omega} b(u)\left(u_{x}+v_{x}\right) w_{x x} w_{x x x} d x+\int_{\Omega} b(u)\left(u_{x x}+v_{x x}\right) w_{x} w_{x x x} d x \\
& +2 \int_{\Omega}(b(u)-b(v)) v_{x} v_{x x} w_{x x x} d x+\lambda \int_{\Omega}(\sigma(u)-\sigma(v)) w_{x x x x} d x=0
\end{aligned}
$$

The integral terms in the equation above, may be estimated as follows:

$$
\begin{aligned}
\left|\int_{\Omega}(a(u)-a(v)) v_{x x x} w_{x x x} d x\right| & \leq C_{1,1}\|w\|_{\infty}\left\|v_{x x x}\right\|_{2}\left\|w_{x x x}\right\|_{2} \\
& \leq K_{1}\left\|w_{x x}\right\|\left\|_{2}\right\| v_{x x x}\left\|_{2}\right\| w_{x x x} \|_{2} \\
& \leq K_{2}\left\|v_{x x x}\right\|_{2}^{2}\left\|w_{x x}\right\|_{2}^{2}+\epsilon_{2}\left\|w_{x x x}\right\|_{2}^{2}, \\
\left|\int_{\Omega} b(u)\left(u_{x x}+v_{x x}\right) w_{x} w_{x x x} d x\right| & \leq\left. C_{2,0}\left\|w_{x}\right\|_{\infty}\left\|u_{x x}+v_{x x}\right\|\right|_{2}\left\|w_{x x x}\right\|_{2} \\
& \leq K_{3}\left\|w_{x x}\right\|_{2}\left\|u_{x x}+v_{x x}\right\|_{2}\left\|w_{x x x}\right\|_{2} \\
& \leq K_{4}\left\|w_{x x}\right\|_{2}^{2}+\epsilon_{2}\left\|w_{x x x}\right\|_{2}^{2} .
\end{aligned}
$$


The inequality obtained by this procedure, for sufficiently small $\epsilon_{2}$, is

$$
\begin{gathered}
\frac{d}{d t}\left\|w_{x x}(t)\right\|_{2}^{2}+c_{*}\left\|w_{x x x}(t)\right\|_{2}^{2} \leq M_{0}(t)\left\|w_{x x}(t)\right\|_{2}^{2}, \\
M_{0}(t)=C_{1}+C_{2}\left\|v_{x x x}(t)\right\|_{2}^{2} .
\end{gathered}
$$

Since the solution $v \in L^{2}\left(0, T ; H^{3} \cap H_{0}^{1}(\Omega)\right)$ (e.g., see Lemma 3.4), the function $M_{0}(t)$ is integrable on the interval $[0, T]$. Therefore, the standard Gronwall lemma is applicable to inequality (3.37a) to obtain

$$
\left\|w_{x x}(t)\right\|_{2}^{2} \leq C_{3}\left\|w_{x x}(0)\right\|_{2}^{2}, \quad C_{3}=\exp \left\{\max _{t \in[0, T]} M_{0}(t)\right\}
$$

Inequality (3.38) implies the continuity of the mapping $S(t): u_{0} \in H \mapsto u(t) \in$ H.

Now, we are allowed to define a dynamical system in $H$ as the mapping

$$
S(t): u_{0} \in H \longmapsto u(t) \in H
$$

associated to problem (1.1). We conclude with the following result.

Theorem 3.6. If $f \in H$, then the semigroup $S(t)$ possesses global attractor $A$ in $H$.

Proof. Restating the result of Lemma 3.3 and taking into account inequality (3.25) for some fixed $r>0$, we have that the closed ball in $H$,

$$
\mathscr{B}_{1}=\left\{\phi \in H:\|\phi\|_{H} \leq \rho_{3}\right\}
$$

is a bounded absorbing set for the semigroup $S(t)$, that is, for every bounded set $\mathscr{B}$ in $H$, there exists $t_{1}(B)>0$, such that $S(t) \mathscr{B} \subset \mathscr{B}_{1}$, for every $t \geq t_{1}(\mathscr{B})$. On the other hand, Lemma 3.4 implies that there exists $t_{2}(\mathscr{B})>0$ such that $S(t) \mathscr{B} \subset B_{2}$ for $t \geq t_{2}(\mathscr{H})$, where

$$
\mathscr{B}_{2}=\left\{\phi \in X:\|\phi\|_{X} \leq \rho_{4}\right\}
$$

is a closed ball in $X:=H^{3} \cap H_{0}^{1}(\Omega)$. The set $\mathscr{B}_{2}$ is bounded in $X$ and relatively compact in $H$ and the semigroup $S(t)$ is uniformly compact. Hence, the set $\mathscr{A}=$ $\omega(\mathscr{B})$ is a compact attractor for the semigroup $S(t)$. 


\section{Acknowledgment}

This work was partially sponsored by a grant from the Research Committee of the National Technical University of Athens (NTUA), Athens, and by a grant from THALES Project of Basic Research Training, NTUA, Athens.

\section{References}

[1] N. D. Alikakos and P. W. Bates, Stabilization of solutions for a class of degenerate equations in divergence form in one space dimension, J. Differential Equations 73 (1988), no. 2, 363-393.

[2] F. Andreu, J. M. Mazón, F. Simondon, and J. Toledo, Attractor for a degenerate nonlinear diffusion problem with nonlinear boundary condition, J. Dynam. Differential Equations 10 (1998), no. 3, 347-377.

[3] D. Aronson, Regularity of flows in porous media: a survey, Nonlinear Diffusion Equations and Their Equilibrium States, I (Berkeley, CA, 1986), Math. Sci. Res. Inst. Publ., vol. 12, Springer-Verlag, New York, 1988, pp. 35-49.

[4] D. Aronson, M. G. Crandall, and L. A. Peletier, Stabilization of solutions of a degenerate nonlinear diffusion problem, Nonlinear Anal. 6 (1982), no. 10, 1001-1022.

[5] R. Balescu, Transport Processes in Plasmas, vol. 2, North-Holland, Amsterdam, 1988.

[6] D. S. Cohen and J. D. Murray, A generalized diffusion model for growth and dispersal in a population, J. Math. Biol. 12 (1981), no. 2, 237-249.

[7] Le Dung, Remarks on Hölder continuity for parabolic equations and convergence to global attractors, Nonlinear Anal. 41 (2000), no. 7-8, Ser. A: Theory Methods, 921-941.

[8] A. Eden, B. Michaux, and J.-M. Rakotoson, Doubly nonlinear parabolic-type equations as dynamical systems, J. Dynam. Differential Equations 3 (1991), no. 1, 87-131.

[9] D. E. Edmunds and W. D. Evans, Spectral Theory and Differential Operators, Clarendon Press, Oxford, 1990.

[10] E. Feireisl, Asymptotic behavior and attractors for degenerate parabolic equations on unbounded domains, Nonlinear Anal. 26 (1996), no. 9, 1535-1546.

[11] E. Feireisl, P. Laurençot, and F. Simondon, Global attractors for degenerate parabolic equations on unbounded domains, J. Differential Equations 129 (1996), no. 2, 239261.

[12] O. A. Ladyzhenskaya, The Boundary Value Problems of Mathematical Physics, Applied Mathematical Sciences, vol. 49, Springer-Verlag, New York, 1985.

[13] M. Langlais and D. Phillips, Stabilization of solutions of nonlinear and degenerate evolution equations, Nonlinear Anal. 9 (1985), no. 4, 321-333.

[14] J. D. Murray, Mathematical Biology, 2nd ed., Biomathematics, vol. 19, SpringerVerlag, Berlin, 1993.

[15] M. Schatzman, Stationary solutions and asymptotic behavior of a quasilinear degenerate parabolic equation, Indiana Univ. Math. J. 33 (1984), no. 1, 1-29.

[16] R. Temam, Infinite-Dimensional Dynamical Systems in Mechanics and Physics, 2nd ed., Applied Mathematical Sciences, vol. 68, Springer-Verlag, New York, 1997.

[17] E. Zeidler, Nonlinear Functional Analysis and Its Applications. I. Fixed-Point Theorems, Springer-Verlag, New York, 1986.

[18] _ Nonlinear Functional Analysis and Its Applications. II/A. Linear Monotone Operators, Springer-Verlag, New York, 1990. 


\section{Semibounded evolution equation}

[19] Nonlinear Functional Analysis and Its Applications. II/B. Nonlinear Monotone Operators, Springer-Verlag, New York, 1990.

Nikos Karachalios: Department of Statistics and Actuarial Science, School of Sciences, University of the Aegean, Karlovassi, 83200 Samos, Greece

E-mail address: karan@aegean.gr

Nikos Stavrakakis: Department of Mathematics, National Technical University, Zografou Campus, 15780 Athens, Greece

E-mail address: nikolas@central.ntua.gr

Pavlos Xanthopoulos: Department of Mathematics, National Technical University, Zografou Campus, 15780 Athens, Greece 


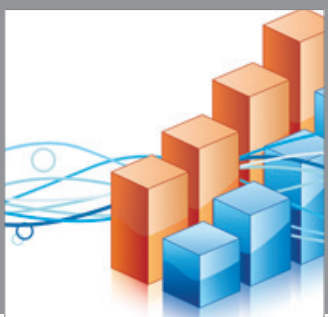

Advances in

Operations Research

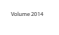

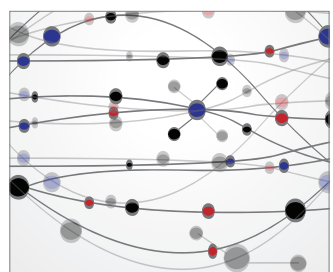

\section{The Scientific} World Journal
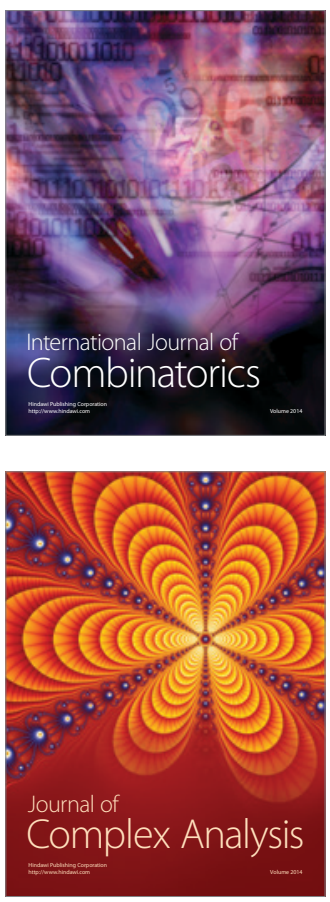

International Journal of

Mathematics and

Mathematical

Sciences
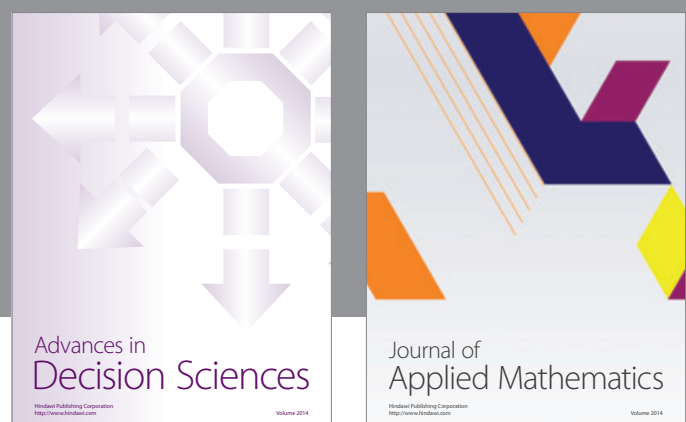

Journal of

Applied Mathematics
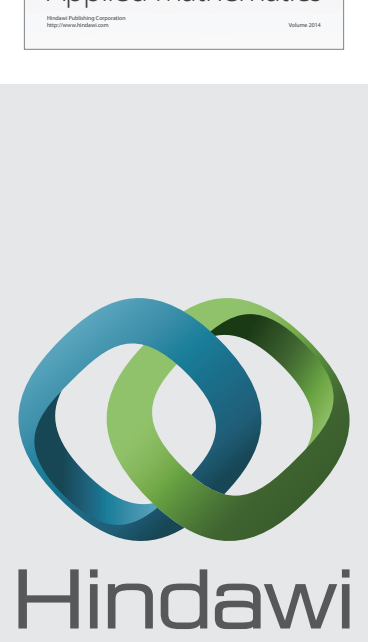

Submit your manuscripts at http://www.hindawi.com
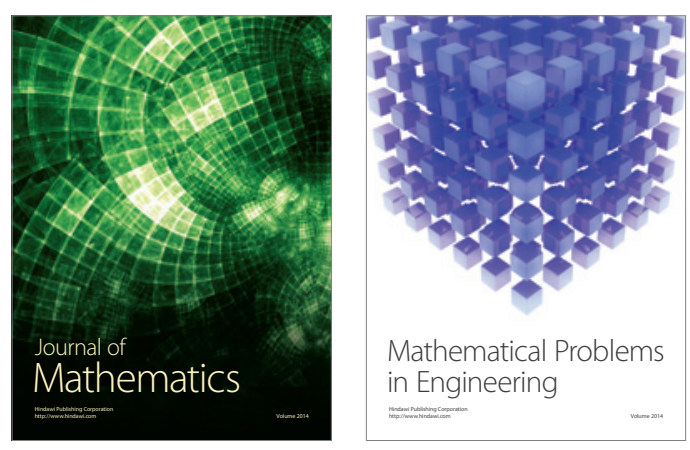

Mathematical Problems in Engineering
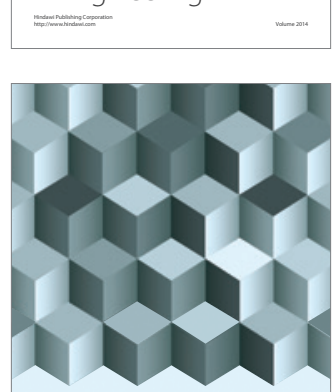

Journal of

Function Spaces
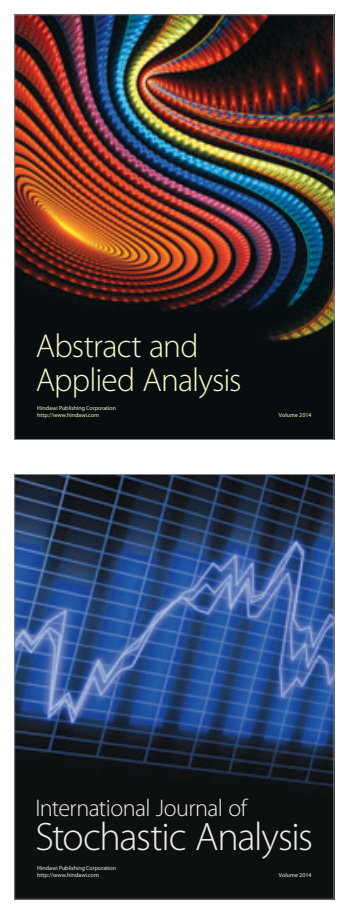

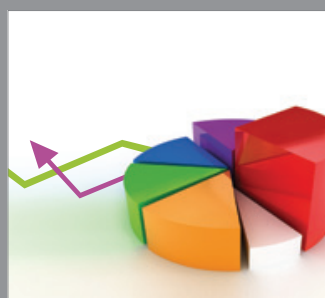

ournal of

Probability and Statistics

Promensencen
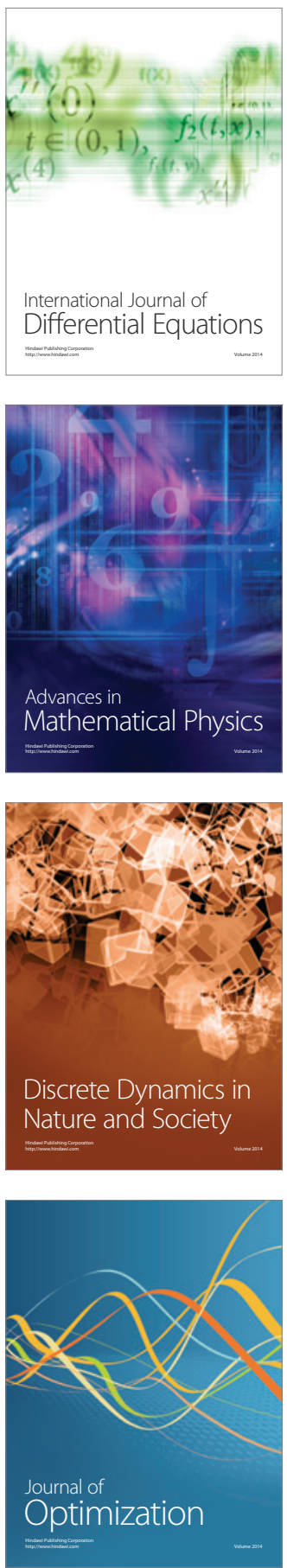